\title{
le portiQue Portique
}

Revue de philosophie et de sciences humaines

$29 \mid 2012$

Georges Bataille

\section{Bataille, entre Kojève et Queneau : le désir et l'histoire}

Bataille, between Kojève and Queneau: history and desire

Bataille zwischen Kojève und Queneau: das Begehren und die Geschichte

\section{Philippe Sabot}

\section{OpenEdition}

Journals

Édition électronique

URL : http://journals.openedition.org/leportique/2594

DOI : $10.4000 /$ leportique.2594

ISSN : $1777-5280$

Éditeur

Association "Les Amis du Portique"

Édition imprimée

Date de publication : 25 octobre 2012

ISSN : 1283-8594

\section{Référence électronique}

Philippe Sabot, «Bataille, entre Kojève et Queneau : le désir et l'histoire », Le Portique [En ligne], 29 |

2012, document 2, mis en ligne le 15 décembre 2014, consulté le 25 mars 2021. URL : http:// journals.openedition.org/leportique/2594; DOI : https://doi.org/10.4000/leportique.2594

Ce document a été généré automatiquement le 25 mars 2021.

Tous droits réservés 


\title{
Bataille, entre Kojève et Queneau : le désir et l'histoire
}

\author{
Bataille, between Kojève and Queneau: history and desire \\ Bataille zwischen Kojève und Queneau: das Begehren und die Geschichte
}

\section{Philippe Sabot}

1 L'importance des leçons sur la Phénoménologie de l'esprit professées par Alexandre Kojève entre janvier 1933 et mai 1939 à l'École des Hautes Études n'est plus à démontrer. Ces leçons marquent bien sûr une étape décisive dans la réception de la pensée hégélienne en France, qui s'est trouvée à partir de là réorientée vers un ouvrage jusque là négligé par la plupart des commentateurs (à part Jean Wahl) et qui devait faire l'objet dans la décennie suivante de toutes les attentions (en particulier à partir de la traduction de la Phénoménologie par Jean Hyppolite au début des années 1940). Mais, l'enseignement de Kojève tire également son importance de l'influence qu'il a manifestement exercée sur toute une génération de penseurs et d'écrivains, pour lesquels il a pu apparaitre, selon les mots de V. Descombes dans Le Même et l'Autre, comme «le point d'intersection des multiples références de l'époque ${ }^{1}:$ marxisme, existentialisme, phénoménologie, psychanalyse ${ }^{2}$, etc. N'oublions pas que les leçons de Kojève ont eu pour auditeurs (plus ou moins assidus) Raymond Queneau, Georges Bataille,Raymond Aron,Roger Caillois,Michel Leiris,Henry Corbin,Maurice MerleauPonty,Jacques Lacan,Jean Hippolyte, Éric Weil... De ce point de vue, on pourrait même dire que l'ouvrage dans lequel l'enseignement de Kojève a été recueilli, son Introduction à la lecture de Hegel (éditée par Raymond Queneau en 1947), fournit encore aujourd'hui une archive privilégiée pour rendre compte du «système de pensée " propre à cette époque. Par "système de pensée ", il faut entendre à la fois la cohérence d'ensemble d'un certain nombre de concepts et de thèmes identifiables (le désir, la reconnaissance, la fin de l'histoire, la sagesse) et un champ de problèmes suscitant le débat et même la polémique dans la période de l'après-guerre (que l'on pense notamment à la discussion avec Léo Strauss concernant l'implication politique du philosophe). C'est dans cette perspective " archéologique » que nous voudrions situer les analyses qui vont suivre en les inscrivant sous l'horizon d'une question générale : comment penser d'après Kojève 
(au sens de sa postérité et de la fécondité de son héritage) ce qui vient après lui (au sens d'une postériorité) ? Nous envisagerons cette question à partir d'un biais particulier qui concerne au premier chef deux des auditeurs les plus assidus de l'enseignement de Kojève, Raymond Queneau et Georges Bataille dont l'œuvre et la pensée ont été profondément marqués par la lecture kojévienne de Hegel mais qui, précisément, élaborent à partir de cette lecture ou d'après cette lecture, des interprétations distinctes, mais peut-être pas complètement incompatibles, des «fins de l'homme». Nous allons donc nous efforcer de montrer en quoi le « kojévisme » définit en quelque sorte ce champ de problèmes communs à partir duquel Bataille et Queneau ont pu chacun faire entendre leur voix propre, en poursuivant d'ailleurs tous deux, quoique selon des modalités différenciées, la voie de l'écriture littéraire - comme si la démarche et le style d'enseignement de Kojève portaient déjà en eux-mêmes, sinon l'exigence, du moins la possibilité de ce débordement du concept par la fiction.

2 Pour bien circonscrire ce champ de problèmes à partir duquel nous envisageons de situer Queneau et Bataille l'un par rapport à l'autre, mais aussi l'un et l'autre par rapport à Kojève, il convient de rappeler d'abord quelques traits saillants de l'interprétation kojévienne de la Phénoménologie de l'esprit de Hegel ${ }^{3}$.

\section{Désir, Sagesse et fin de l'Histoire}

L'ensemble de la lecture kojévienne de Hegel s'ordonne à une polarité fondamentale des expériences de la conscience, qui oppose globalement le Désir et la Sagesse, selon un dispositif théorique (et rhétorique) qui tend à reconstruire complètement la Phénoménologie hégélienne à partir de deux de ses «moments " privilégiés : d'une part, la Section A du chapitre IV de la Phénoménologie («Autonomie et dépendance de la conscience de soi : Maîtrise et servitude ») - dont la "traduction commentée », parue une première fois dans la revue Mesures en janvier 1939, est reprise «en guise d'introduction » dans l'Introduction à la lecture de Hegel; et d'autre part, le chapitre VIII de la Phénoménologie où se trouve développée la perspective du «Savoir absolu » et qui, dans l'interprétation de Kojève, sert à lier le destin historique de l'Homme (sa fin, au sens de sa disparition) à la perspective d'une Sagesse post-historique (qui correspond à la fin de l'Homme, au sens de son achèvement) ${ }^{4}$. Cette polarisation historique de l'expérience humaine entre Désir et Sagesse se fonde en réalité sur la tension entre « insatisfaction » et « satisfaction ».

5 Kojève place au principe de son interprétation de Hegel la thématique d'un Désir anthropogène. C'est ce qui apparaît de manière évidente dans l'interprétation que Kojève donne $\mathrm{du}$ passage sur la «dialectique du maître et de l'esclave». Cette interprétation, qui forme la matrice conceptuelle de l'hégélianisme hétérodoxe de Kojève, s'alimente aussi bien à une doctrine de la finitude reprise de Heidegger qu'à une perspective historico-politique héritée d'une certaine vulgate marxiste. De la «traduction commentée » proposée à l'ouverture de l'Introduction, ressort en effet d'abord l'idée d'une négativité fondamentale du désir qui trouve à s'exprimer pleinement dans le rapport non pas à l'être-donné dans la Nature, mais dans la confrontation avec un autre désir. L'analyse de la « réalité-humaine » repose alors sur une ontologie dualiste qui pose le "déchirement du Réel en Homme et en Nature » 
comme le fondement de la dialectique sociale de la reconnaissance, présentée d'emblée comme une dialectique des désirs. Tant qu'il s'en tient à l'ordre d'un désir seulement naturel qui porte sur la vie, l'homme est en effet incapable d'accéder à la conscience de soi, donc à l'humanité en tant que telle et à l'ordre historique qui en soutient le déploiement ontologique. Inscrit dans l'être-donné et à partir de lui (la Nature), le désir naturel, de l'ordre du simple besoin, est condamné à éprouver sa mauvaise infinité et renvoie le Moi du désir à sa propre animalité. L'accès à la conscience de soi suppose donc que le désir porte sur un objet non-naturel, "autre chose qu'une chose » qui dépasse le donné immédiat, c'est-à-dire sur le désir lui-même, sur le désir en tant que tel, dans sa structure de désir, c'est-à-dire "avant sa satisfaction ». Ce désir n'est en effet rien d'autre qu'une béance, qu'un trou dans l'être, qu'un "vide irréel » et " avide ", " présence de l'absence d'une réalité » ${ }^{5}$ en l'homme, donc la révélation d'un «néant » qui doit encore s'avérer pour dépasser la simple contemplation anxiogène de cet état. Ainsi, en se "nourrissant » de désir, l'homme s'apparaît à lui-même comme désir; son être se fait Action, "négativité-négatrice " de soi comme être naturel et transformation du donné « en ce qui n'était pas là avant » :

Son maintien dans l'existence signifiera donc pour ce Moi : «ne pas être ce qu'il est (en tant qu'être statique et donné, en tant qu'être naturel) et être (c'est-à-dire devenir) ce qu'il n'est pas». Ce Moi sera ainsi son propre œuvre: il sera (dans l'avenir) ce qu'il est devenu par négation (dans le présent) de ce qu'il a été (dans le passé), cette négation étant effectuée en vue de ce qu'il deviendra. Dans son être même, ce Moi [...] est l'acte de transcender ce donné qui lui est donné et qu'il est lui-même ${ }^{6}$.

Nous voyons dans ces quelques lignes comment Kojève aménage, au sein de sa propre interprétation du texte hégélien, une place pour son interprétation de la finitude heideggérienne, ramenée elle aussi sur un plan strictement anthropologique ${ }^{7}$. En effet, le désir humain consiste à introduire dans la plénitude immédiate de sa vie seulement animale, la perspective de ce moment à venir que constitue la possibilité de ne plus être. L'homme "néantise » le présent (comme succession de "maintenant») ou s'en absente, et se projette ainsi dans l'indétermination du futur. La finitude creuse le désir humain d'une insatisfaction chronique qui est la marque de son projet. Or, la description phénoménologique du désir anthropogène ne s'en tient pas à cette expérience de la finitude qui soutient la structure néantisante du projet proprement humain, distingué de la simple vie animale. En effet, pour se saisir réflexivement, s'avérer comme négativité-négatrice dans une Histoire, le désir doit s'aliéner, passer par l'épreuve d'un autre désir. C'est ici que Marx rejoint Heidegger dans la lecture kojévienne de Hegel. Notons en passant que cette convergence, à première vue étonnante mais tout à fait dans le style de Kojève, est justifiée par ce dernier dans une note, où il écrit que «Heidegger a repris les thèmes hégéliens de la mort; mais il néglige les thèmes complémentaires de la Lutte et du Travail ; aussi sa philosophie ne réussit-elle pas à rendre compte de l'Histoire. Marx maintient les thèmes de la Lutte et du Travail, et sa philosophie est essentiellement "historiciste"; mais il néglige le thème de la mort (tout en admettant que l'homme est mortel) ${ }^{8}$. Kojève tire de cette réflexion l'idée que le mouvement qui institue non seulement l'Humain comme " négativité-négatrice ", mais encore l'Histoire humaine comme " histoire des désirs désirés $"{ }^{9}$, consiste bien à vouloir posséder l'autre dans son désir, l'autre en tant que désir et à se l'assimiler comme tel. 
7 Kojève prend donc appui sur la fameuse séquence de la lutte pour la reconnaissance pour développer sa propre théorie de l'homme historique comme sujet néantisant, qui exerce sa négativité essentielle à travers les formes conjointes de la Lutte et du Travail. On connaît bien le processus, mis en exergue par Kojève, suivant lequel la conscience de soi ne peut se manifester qu'en entrant dans ce fatal et paradoxal "doublement " (qu'incarnent les figures concurrentes du Maître et de l'Esclave) qui va la faire dépendre de cette autre conscience de soi (de cet autre désir) dont elle veut pourtant se faire reconnaître dans sa réalité transcendante de pur désir indépendant. Ainsi, en faisant de ce processus l'épure de l'institution anthropologico-historique de l'humain, Kojève peut écrire que le maitre est «le catalyseur du processus historique, anthropogène " ${ }^{10}$ - processus que néanmoins l'esclave met en œuvre effectivement, en se livrant à l'activité négatrice et transformatrice du donné qui constitue immédiatement sa servitude mais qui lui révèle médiatement sa propre finitude néantisante, donc son humanité: "L'histoire de l'homme est l'histoire de son travail ${ }^{11}$.

8 Or, cette phénoménologie du désir anthropogène, que Kojève élabore à partir de Hegel (et à vrai dire surtout en en partant, en s'en écartant), appelle comme son complément une doctrine de la Sagesse qui se fonde sur une autre dialectique, rejouant sur un tout autre plan la dialectique de la reconnaissance, puisqu'elle cherche à penser, à partir des conférences consacrées au "Savoir absolu », l'articulation de l'Histoire et de l'Absolu, ou encore de l'insatisfaction et de la satisfaction. En effet, il y a bien Histoire aussi longtemps que l'Homme est en proie à l'insatisfaction du Désir, aussi longtemps donc qu'il est engagé dans ce processus de négativité-négatrice dont témoigne de manière exemplaire la lutte pour la reconnaissance. Mais, ce processus, qui co-implique l'Histoire et l'Homme, a lui-même une fin. Kojève le souligne dès son interprétation de " Maîtrise et servitude ", avant d'y revenir dans ses dernières conférences :

Si l'homme n'est pas autre chose que son devenir, [...] si la réalité révélée n'est rien d'autre que l'histoire universelle, cette histoire doit être l'histoire de l'interaction entre Maîtrise et Servitude : la "dialectique» historique est la "dialectique du Maître et de l'esclave ». Mais si l'opposition de la «thèse» et de l' "antithèse " n'a un sens qu'à l'intérieur de la conciliation de la "synthèse ", si l'Histoire au sens fort du mot a nécessairement un terme final, si l'homme qui devient doit culminer en l'homme devenu, si le Désir doit aboutir à la satisfaction, [...] l'interaction du maître et de l'esclave doit finalement aboutir à leur "suppression dialectique » ${ }^{12}$.

On voit sur quelles bases fragiles (thèse-antithèse-synthèse) s'opère ici la « relève » de l'Homme historique. Kojève superpose au thème anthropologique du désir insatisfait, de la négativité propre au désir humain comme désir d'un être fini, le thème d'une négation de cette négativité qui ne peut s'accomplir que sous la double condition d'une fin de l'Histoire et d'un anéantissement du sujet désirant lui-même. La libération du désir constitue ainsi le sens de l'histoire, sa finalité et son terme. Le "Savoir absolu " représenterait ainsi ce moment terminal de l'humanité et du monde historique où peuvent (enfin) coïncider conscience de soi et satisfaction. Le Sage, dont Hegel luimême constitue aux yeux de Kojève l'incarnation exemplaire, "réalise " le savoir absolu en sa propre personne et en son propre Livre, la Phénoménologie de l'esprit, où la réalité humaine récapitule son sens, tel qu'il a pu s'effectuer concrètement dans l'action elle-même exemplaire du dernier héros historique (Napoléon). Voici comment Kojève rend compte de cette auto-suppression de l'Homme historique qui ouvre la voie au « Savoir absolu », et à la Sagesse : 
L'Homme est donc un Néant qui néantit et qui ne se maintient dans l'Être qu'en niant l'être, cette Négation étant l'Action. Or si l'Homme est Négativité, c'est-à-dire Temps, il n'est pas éternel. Il naît et il meurt en tant qu'Homme. Il est « das Negative seiner Selbst », dit Hegel. Et nous savons ce que cela signifie : l'Homme se supprime en tant qu'Action (ou Selbst) en cessant de s'opposer au Monde, après y avoir créé l'État universel et homogène; ou bien, sur le plan cognitif : l'Homme se supprime en tant qu'Erreur (ou «Sujet» opposé à l'objet) après avoir créé la Vérité de la «Science ${ }^{13}$. propre essence comme négativité, fixe les conditions d'une nouvelle vie pour les hommes de la Post-Histoire: cette vie n'est plus commandée par le désir de reconnaissance et par la négativité qui l'avait poussé à s'accomplir dans la lutte sociale et par le travail; elle est une existence désormais soumise à la seule loi de la «Befriedigung » (Satisfaction), et consacrée, comme le dit Kojève lui-même, à toutes ces activités déliées de toute préoccupation d'ordre empirique : «l'art, l'amour, le jeu, etc., etc. ; bref, tout ce qui rend l'Homme heureux $»{ }^{14}$.

11 Cette récapitulation d'ensemble de la lecture kojévienne de Hegel permet donc de mettre en évidence ce qui se trouve en débat chez Bataille et chez Queneau et d'une certaine façon entre Bataille et Queneau - à savoir précisément cette polarité du Désir et de la Sagesse dont ils proposent des lectures très différentes mais non nécessairement divergentes, voire complémentaires.

\section{La négativité sans emploi}

Bataille a été un auditeur passionné des cours de Kojève dont selon ses propres termes il est souvent sorti « rompu, broyé, tué dix fois » ${ }^{15}$. Ces cours, auxquels il a assisté aux côtés de Queneau, ont sans doute contribué à modifier en profondeur sa conception, ou plutôt sa perception de la pensée hégélienne, telle qu'elle était exposée dans un article de La Critique sociale daté de 1932 et co-signé avec Raymond Queneau justement : «La critique des fondements de la dialectique hégélienne ». Cette « critique » dont il s'avère que Bataille a été le rédacteur principal, s'appuyait principalement sur une lecture des travaux de Nicolaï Hartmann et en particulier sur un long article paru en 1931 dans la Revue de Métaphysique et de Morale consacré à « Hegel et le problème de la dialectique du réel ». Elle portait pour l'essentiel sur la question du statut de la dialectique et sur l'opposition entre l'usage idéaliste de la dialectique chez Hegel (selon lequel la dialectique apparaît "sans fondement dans la réalité » ${ }^{16}$ ) et son usage matérialiste et politique qui se joue "sur le terrain immédiat de la lutte des classes, dans l'expérience, et non dans les nuées aprioriques des conceptions universelles $"{ }^{17}$. De ce point de vue, l'interprétation kojévienne de la dialectique du maître et de l'esclave qui a constitué un temps fort de l'enseignement dispensé à l'École des Hautes Études ne devait pas manquer d'impressionner fortement Bataille en lui présentant un autre Hegel que celui qu'il avait pu fréquenter jusque là de seconde main, à travers les travaux d'histoire de la philosophie de Georges Gurvitch ${ }^{18}$ et de Koyré (il avait été l'auditeur des cours sur la "philosophie religieuse de Hegel» au tout début des années 1930). Néanmoins, et quelle qu'ait pu être la fascination exercée par Kojève sur ses auditeurs, il semble que Bataille a très vite dessiné à partir de son enseignement une trajectoire de pensée originale et singulière, qui devait le conduire par la suite à disjoindre l'expérience, ou $\mathrm{du}$ moins une certaine forme d' expérience vécue par chaque être humain " (selon 
l'expression utilisée à plusieurs reprises dans l'article de $1932{ }^{19}$ ) de toutes les formes prises par sa dialectisation historique.

13 Ce qui est dès lors en jeu, c'est une contestation interne du savoir absolu et donc du schéma kojévien de la mort de l'homme à la fin de l'Histoire. De ce point de vue, il est possible de dire que Bataille en vient à opposer en quelque sorte à l'insatisfaction par défaut proposée par Kojève (comme cette insatisfaction d'avant la satisfaction parfaite du savoir absolu et de la fin de l'histoire) une forme d'insatisfaction par excès qui prend pour nom « négativité sans emploi » ou encore « expérience intérieure ». De quoi s'agitil ? Pour le comprendre, et pour mesurer exactement ce qui sépare Bataille de Kojève (tenu alors pour un interprète fidèle de la pensée hégélienne), il faut citer cet extrait bien connu d'une lettre adressée par Bataille à Kojève le 6 décembre 1937, à la suite de la conférence que Kojève avait donnée deux jours auparavant devant le Collège de Sociologie (sous le titre : « Les conceptions hégéliennes ») - conférence dont il ne reste aucune trace ${ }^{20}$ :

J'admets (comme une supposition vraisemblable) que dès maintenant l'histoire est achevée [...]. Mon expérience, vécue avec beaucoup de souci, m'a conduit à penser que je n'avais plus rien «à faire ». [Or] si l'action (le « faire») est - comme dit Hegel - la négativité, la question se pose alors de savoir si la négativité de qui n'a " plus rien à faire» disparaît ou subsiste à l'état de «négativité sans emploi»: personnellement, je ne puis décider que dans un sens, étant moi-même exactement cette "négativité sans emploi » (je ne pourrais me définir de façon plus précise). [...] J'imagine que ma vie - ou son avortement, mieux encore, la blessure ouverte qu'est ma vie - à elle seule constitue la réfutation du système fermé de Hegel ${ }^{21}$.

La réflexion de Bataille, en première personne, s'articule explicitement au récit kojévien de la fin de l'Histoire. Si le Sage réalise le savoir absolu, cet achèvement correspond bien à l'accomplissement intégral du désir (c'est-à-dire à la «fin » de l'Homme historique). Mais dans la perspective de Bataille, la Sagesse, loin de satisfaire le Désir une fois pour toutes, tend au contraire à l'exacerber au-delà de toute limite, audelà de la fin de l'histoire. Car ce désir, une fois que tout est accompli, n'a plus rien à désirer que ce rien lui-même, sa propre négativité désormais inemployée dans le monde post-historique et n'affectant plus aucun sujet humain dans son autoconstitution. On voit donc qu'au schéma hégéliano-marxiste imaginé par Kojève qui revenait à accorder au négatif un rôle moteur dans la dialectique historique de la reconnaissance, Bataille tend à superposer le thème d'une pure négativité selon lequel la vie se résout en activité en pure perte, sans ancrage positif et sans œuvre à réaliser.

Se dessine donc, dans les marges du kojévisme, la possibilité d'un revers de la dialectique, d'une forme de négativité non dialectisable qui engage l'homme au-delà de son propre achèvement (comme Sagesse) et même dans la contestation de cet achèvement même. Dans le long commentaire qu'il propose du bref commentaire de Bataille à la conférence de Kojève, Maurice Blanchot explicite le sens fondamental de cet inachèvement dans l'achèvement, qui ouvre dans le savoir absolu l'espace du « nonsavoir ", de l'ordre d'une expérience-limite :

Nous supposons l'homme en son essence satisfait; il n'a, homme universel, plus rien à faire, il est sans besoin, il est, même si individuellement il meurt encore, sans commencement, sans fin, en repos dans le devenir de sa totalité immobile. L'expérience-limite est celle qui attend cet homme ultime, capable une dernière fois de ne pas s'arrêter à cette suffisance qu'il atteint ; elle est le désir de l'homme sans désir, l'insatisfaction de celui qui est satisfait « en tout » [...]. L'expérience-limite est l'expérience de ce qu'il y a hors de tout, lorsque le tout exclut tout dehors, de ce 
qu'il reste à atteindre, lorsque tout est atteint, et à connaître, lorsque tout est connu : l'inaccessible même, l'inconnu même ${ }^{22}$. la perspective kojévienne d'une Sagesse post-historique le thème d'un érotisme transgressif qui exacerbe la relation de l'homme au sacré, en maintenant ouverte, vivante, la dimension d'une existence vouée à l'extrême, à l'expérience et à la diction de l'extrême. En ce sens, dans L'Expérience intérieure (1943), Bataille réinterprète l'hégélianisme (comme pensée du système et pensée systématique) du point de vue de l'expérience, et comme sa dénégation même - de l'ordre d'un refoulement mutilant :

Petite récapitulation comique. - Hegel, je l'imagine, toucha l'extrême. Il était jeune encore et crut devenir fou. J'imagine même qu'il élaborait le système pour échapper (chaque sorte de conquête, sans doute, est le fait d'un homme fuyant une menace). Pour finir, Hegel arrive à la satisfaction, tourne le dos à l'extrême. La supplication est morte en lui. Qu'on cherche le salut, passe encore, on continue de vivre, on ne peut être sûr, il faut continuer de supplier. Hegel gagna, vivant, le salut, tua la supplication, se mutila. Il ne resta de lui qu'un manche de pelle, un homme moderne. Mais avant de se mutiler, sans doute, il a touché l'extrême, a connu la supplication : sa mémoire le ramène à l'abîme perçu, pour l'annuler ! Le système est l'annulation ${ }^{26}$. 
Après avoir reçu le livre de Bataille, Kojève formulera un jugement sévère sur son contenu, qui rappelle celui que Sartre devait porter au même moment dans son étude «Un nouveau mystique ${ }^{27}$ :

Cette critique de Hegel n'est pas une critique de l'hégélianisme qui vit chez MarxLénine-Staline. Pour ceux-ci, la «satisfaction» est dans l'avenir. Donc, pour eux, « on continue à vivre, il n'est pas possible d'être en sûreté, il faut continuer à... »vous dites «supplier». Eux disent «lutter». Voilà toute la différence entre vous et eux. Mais ne dites pas qu'ils ne sont qu'un «manche de pelle». Hegel pensait l'être. Mais Staline est une pelle faite et achevée, qui accomplit très bien sa tâche ${ }^{28}$.

Au-delà de ce correctif visant à réinscrire l'hégélianisme dans l'histoire et où apparaît un clivage profond entre Bataille et Kojève, on notera qu'en opposant au système du sens, et au discours qui le boucle sur lui-même, l'ouverture menaçante de l'expérience, synonyme de risque et d'insatisfaction mais porteuse finalement des fondements existentiels de la réalité humaine, Bataille anticipe à sa manière l'interprétation de Jean Hyppolite qui cherchera à déceler cette opposition, sous la forme d'une tension, au sein même du développement de la pensée hégélienne ${ }^{29}$.

\section{L'histoire, comme pour rire...}

21 Mais tournons, nous aussi, à présent, « le dos à l'extrême » et demandons-nous pour finir si, avec Queneau, le kojévisme ne fait pas les frais d'une "contestation » plus nuancée mais non moins probante fondée notamment sur la reprise décalée de la polarité du Désir et de la Sagesse dans le contexte de fictions littéraires où la conception kojévienne de l'histoire se trouve ironisée et finalement problématisée.

Dans l'article de la revue Critique qu'il a consacré en mai 1952 à quelques romans de son ami Raymond Queneau (Pierrot mon ami (1942), Loin de Rueil (1945) et Le Dimanche de la vie (1952)), Kojève a suggéré qu'il s'agissait précisément de trois «romans de la sagesse ", illustrant donc le bonheur paradoxal de l'Homme post-historique, à travers des personnages qui n'ont littéralement plus rien à faire qu'à promener leur ennui et à raconter des histoires à dormir debout - comme pour achever, non sans dérision, de tuer le temps. Pierre Macherey a déjà longuement analysé le rapport de Queneau à Kojève dans À quoi pense la littérature $?^{30}$ Nous ne reprendrons pas ici le détail de ces analyses mais nous souhaitons insister sur un point important qui concerne la manière dont Queneau a pu reprendre à son compte la doctrine kojévienne de la Sagesse, tout en la prolongeant et en la corrigeant sensiblement en faisant jouer en quelque sorte l'une contre l'autre la fiction et la réalité, comme l'indique d'ailleurs l'avertissement du Dimanche de la vie: «Les personnages de ce roman étant réels, toute ressemblance avec des individus imaginaires serait fortuite ». Ce roman de 1952 auquel Kojève consacre l'essentiel de son article est décrit par ce dernier comme un récit «humoristique d'allure anodine ${ }^{31}$ où il peut sembler étrange, voire complètement saugrenu, de chercher une quelconque trace de philosophie (hégélienne ou autre). Mais on peut aussi bien dire qu'en bonne doctrine kojévienne, ce défaut apparent de "philosophie " est en réalité l'une des manifestations de la « Sagesse » qu'incarne ici, pour les besoins de la cause romanesque, l'indolent Valentin Brû, un soldat démobilisé reconverti dans le petit commerce (il tient une boutique de cadres pour photographies) et marié (presque malgré lui) à la vigoureuse Julia.

Valentin Brû donne une image assez désolante, et néanmoins amusante, en tout cas décalée et inattendue, du Sage kojévien qui se caractérise, selon Kojève lui-même, par 
«la parfaite satisfaction accompagnée d'une plénitude de la conscience de soi ${ }^{32}$. Or, si Brû semble effectivement pleinement satisfait et n'a manifestement plus rien à attendre de la vie et de ses aléas, surtout depuis qu'il a réalisé le voyage de ses rêves sur le champ de bataille d'Iéna où Hegel avait aperçu en 1807 l'Âme du monde, Napoléon, cette satisfaction confine le plus souvent à l'hébétude et à une profonde indifférence à l'égard de la réalité temporelle et historique - comme si, pour lui, le temps s'était effectivement arrêté et qu'il n'avait plus rien à désirer ni donc à attendre d'une Histoire vouée pour l'essentiel à la répétition sans fin du passé. La «sagesse » du soldat Brû correspond donc à cette plénitude paradoxale de la conscience de soi qui coïncide avec sa vacuité la plus totale: le Sage, parfaitement satisfait, est devenu comme étranger aux luttes qui mettent aux prises ses contemporains et dont il peut interpréter le sens et la nécessité d'un point de vue détaché, celui précisément du savoir absolu. Ce détachement du Sage conduit Valentin Brû à exercer, outre sa profession de vendeurs de cadres (destinés à fixer les souvenirs des autres), la délicate profession de voyante, diseuse de bonne aventure - étant entendu que, de son point de vue, toute aventure, bonne ou mauvaise, est accomplie et ne mérite d'être (re)dite qu'à ceux qui sont insatisfaits de leur vie actuelle et qui désirent en changer - sans savoir donc que c'est impossible. D'où cet échange savoureux que Valentin, exerçant ses talents de voyante sous le nom et l'apparence de Madame Saphir, a avec l'une de ses premières clientes :

La première cliente fut une jeune femme.

- Vous allez vous marier avant peu, dit Valentin, d'une petite voix de fausset qui

faillit le faire rire.

- Je suis mariée depuis 8 jours, dit la consultante.

- C'est bien ce que je disais. Le temps ne compte pas pour nous autres. Hier, demain, qu'est-ce que c'est en face de l'Éternité ?

Si je lui en fous pas plein la vue avec ça, se dit Valentin assez satisfait de son éloquence. Mais la fille était coriace :

- Madame, l'Éternité, c'est bien joli, mais pour moi ce qui compte, c'est pas hier,

c'est demain.

- Hier compte aussi, dit Valentin. Sans hier, demain n'existerait pas.

- En tout cas, je suis mariée depuis 8 jours.

- Que désirez-vous savoir?

- Tout.

- C'est beaucoup, dit Valentin, d'une voix lugubre.

- Madame, je paierai ce qu'il faudra ${ }^{33}$.

Heureusement pour Valentin, la « consultante » s'assoupit pendant qu'il réfléchit à ce qu'il va pouvoir lui raconter et il peut lui extorquer dix francs en lui faisant croire qu'il lui a « tout » dit. En réalité, il ne lui a dit que ce qu'elle savait déjà mais où est contenu au fond tout ce qu'il y a à savoir dès lors que l'on se place au point de vue de l'éternité, c'est-à-dire hors du temps orienté des désirs et de l'insatisfaction, au-delà de l'attente d'une nouveauté imprévisible. La plénitude de la conscience de soi a donc comme condition de possibilité la vacuité même du temps qui passe sans que plus rien ne s'y passe que ce qui s'est déjà passé.

Pourtant, Queneau ne présente pas seulement dans son roman cette version caricaturale de la Sagesse kojévienne. Ou du moins ne manque-t-il pas de confronter cette sagesse à la réalité historique du deuxième conflit mondial. Valentin Brû a bien prévu que la guerre allait éclater, puisqu'après tout, sa nécessité est inscrite dans le cours d'une histoire toujours déjà accomplie et selon lui achevée. Mais il s'est manifestement trompé sur son issue et, après avoir été à nouveau démobilisé au moment de l'armistice de 1940, il renonce à une Sagesse illusoire parce que finalement 
déconnectée de l'histoire pour renouer avec les préoccupations empiriques et même charnelles de la vie réelle, à côté de laquelle il était passé en croyant qu'il pouvait désormais s'en passer. Le désœuvrement du Sage Brû laisse place dans la scène finale du roman à cette "bonne humeur " et à cette joie spontanée qui caractérise selon Hegel, cité en épigraphe du roman ${ }^{34}$, le « dimanche de la vie » - à l'opposé de l'apathie et de la réflexivité maladives à laquelle Valentin Brû s'était livré auparavant, lorsqu'il était persuadé que l'Histoire était finie.

Valentin regardait cette comédie [il s'agit du spectacle donné par les nombreux voyageurs qui essayent de se glisser dans le train qui retourne vers Paris], impassible et immobile. Julia ne réagit pas tout de suite, puis elle faillit l'appeler, mais elle se retint. Valentin, en effet, venait de se mettre en mouvement et de commencer une savante manœuvre. Trois jeunes filles, inexplicablement habillées en alpinistes, profitaient de la décence de ce costume pour essayer de grimper dans un compartiment par la fenêtre. Valentin s'était approché d'elles pour les aider aimablement dans leur entreprise. Julia s'étouffa de rire : c'était pour leur mettre la main aux fesses ${ }^{35}$.

Valentin quitte clairement l'impassibilité et même l'immobilité du Sage (qui est celle de l'Histoire elle-même dès lors qu'elle est accomplie) pour rejoindre le camp des "philosophes", ainsi que sont nommés ces voyeurs qui, au Palace de la Rigolade dans Pierrot mon ami, s'arrangent pour reluquer, sans avoir l'air d'y toucher, les dessous des filles occupées à profiter des attractions de la fête foraine. En tout cas, pour embrayer sur une métaphore que semble appeler la fin du roman de Queneau, le soldat Brû renoue in fine avec le "train » de l'histoire et son lot de rencontres imprévisibles et il renonce définitivement «à tuer le temps et [à] balayer en lui les images d'un monde que l'Histoire allait éponger ${ }^{36}$.

Queneau n'envisage pas, à la manière de Sartre, cette remobilisation finale de son personnage sous la forme d'un «engagement " (Valentin Brû est l'anti-Goetz, le personnage principal du Diable et le Bon Dieu [1951]). Il s'agit plutôt d'un dégagement, d'un pied de nez ironique à une figure de la Sagesse et à une thématique de la « fin de l'histoire » qui, prises au pied de la lettre, sont soit terrifiantes soit ridicules, ou au moins dérisoires - et le plus souvent les deux à la fois. Il est intéressant de souligner de ce point de vue que Kojève, dans son article sur Queneau, a aperçu cette difficulté liée à l'interprétation de sa propre pensée :

Du point de vue hégélien, tant que ne sont pas terminées les dernières luttes négatrices et les derniers travaux transformateurs du donné, indispensables à la création d'un monde où l'homme pourrait justifier à ses propres yeux et aux yeux de tous ses loisirs pacifiques, c'est-à-dire tant que dure l'Histoire proprement dite, la quiétude sans histoire ne peut être qu'un simulacre de la sagesse qui, parfois l'imite il est vrai à s'y méprendre ${ }^{37}$.

En transposant dans l'ordre d'un récit de fiction, la figure du Sage kojévien, Queneau en a effectivement produit un "simulacre». Mais cette simulation imaginaire vient éclairer d'un jour nouveau et ironique la fiction kojévienne de la Sagesse, adossée à une conception héroïque de la fin de l'Histoire que les tribulations loufoques et dérisoire de Valentin Brû contribuent fortement, et... drôlement, à relativiser.

Concluons. Ce qui caractérise en somme ces deux voies du "kojévisme» qu'ont empruntées tour à tour Bataille et Queneau, c'est moins l'accent mis sur le Désir ou sur Sagesse dans la perspective d'une réflexion sur la fin de l'histoire ou sur l'expérience 
humaine, que la capacité à feindre de prendre au sérieux les spéculations théoriques élaborées par Kojève pour rendre problématique, et peut-être dérisoire, le "point de vue hégélien " dont elles se revendiquent. Dans cette perspective, il semble qu'à la différence de Queneau, Bataille a cru que Kojève pensait tout ce qu'il disait sans mesurer la part de fiction et d'imagination qui entrait nécessairement dans la tentative post-hégélienne d'en finir avec l'Histoire.

\section{NOTES}

1. . Vincent Descombes, Le Même et l'Autre. Quarante-cinq ans de philosophie française (1933-1978), Paris, Minuit, 1979, p. 23.

2. . On peut rappeler que MERLEAU-PONTY écrira dans Sens et non-sens : « Hegel est à l'origine de tout ce qui s'est fait de grand en philosophie depuis un siècle... » (Paris, Nagel, 1948, p. 109).

3. . Signalons, concernant Kojève, un regain d'intérêt dans la période récente pour son œuvre et sa pensée. Au travail monographique pionnier de Dominique Auffret, Alexandre Kojève.La philosophie, l'État, la fin de l'Histoire (Grasset \& Fasquelle, 1990), sont venues s'ajouter récemment les études de Dominique Pirotte (Alexandre Kojève. Un système anthropologique, PUF, 2005) et de Marco Filoni (Le Philosophe du dimanche. La vie et la pensée d'Alexandre Kojève, Gallimard, 2010 pour la traduction française).

4. . Nous avons déjà abordé pour elle-même la question de la « fin » de l'homme chez Kojève et chez Foucault dans notre article « De Kojève à Foucault. La « mort de l'homme » et la querelle de l'humanisme » (Archives de philosophie, Cahier 72-3, juillet-septembre 2009, p. 523-540).

5. . Alexandre KоJÈVE, Introduction à la lecture de Hegel, Paris, Gallimard, 1947, p. 12.

6. . Ibid., p. 12-13.

7. . Voir ici les analyses de Dominique PIROTTE, op. cit., chapitre III.

8. . AlexandreкоJÈvE, op. cit., p. 575, note 1.

9. .Ibid., p. 13.

10. .Ibid., p. 30.

11. .Ibid.

12. .Ibid., p. 16.

13. Ibid., p. 435.

14. Ibid., p. 435, note 1 .

15. . Georges BatallLe, Cuvres Complètes, tome VI, Paris, Gallimard, 1973, p. 146, Note.

16. . Georgesbataille, «La critique des fondements de la dialectique hégélienne » (co-écrit avec Raymond Queneau), Cuvres Complètes, tome I, Paris, Gallimard, 1970, p. 278.

17. Ibid., p. 280.

18. . Bataille renvoie notamment à l'ouvrage de Georges GURviTcH intitulé Les Tendances actuelles de la philosophie allemande (Paris, Vrin, 1930).

19. . Georges BATAILle, « La critique des fondements de la dialectique hégélienne » (co-écrit avec Raymond Queneau), op. cit., p. 288.

20. . Dans l'édition des conférences du Collège de sociologie, Denis Hollier a proposé néanmoins une reconstruction de cette conférence.

21. .Georgesbataille, Choix de lettres. 1917-1962, Paris, Gallimard, «Les Cahiers de la NRF », 1997, p. 131-132. 
22. . Maurice BLANCHOT, L'Entretien infini, Paris, Gallimard, 1969, p. 304-305.

23. . Alexandre KoJÈVE, « Préface à l'œuvre de Georges Bataille » [1950], L'Arc, n 44, 1971, p. 36.

24. . Maurice BLANCHOT, op. cit., p. 308, note.

25. . Michel FoucAult, «Préface à la transgression » [1963], Dits et écrits, Paris, Gallimard, 1994, tome 1, p. 235.

26. . Georges Bataille, L’Expérience intérieure, Paris, Gallimard, 1943 ; rééd. Gallimard/Tel, p. 56.

27. . Jean-Paul Sartre, « Un nouveau mystique » [Décembre 1943], in Situations I, Paris, Gallimard, 1947, p. 174-229.

28. . Lettre de Kojève à Bataille [juillet 1943], Textures, n6, 1970, p. 62-63 (citée in Marco Filoni, op. cit., p. 259).

29. . Sur l'hégélianisme de Jean Hyppolite, voir les analyses d'Andrea Bellantone dans Hegel en France, volume 2 : de Vera à Hyppolite, Paris, Hermann, « Philosophie », 2011, p. 275-286.

30. . Pierre MACHEREY, "Divagations hégéliennes de Raymond Queneau ", in À quoi pense la littérature? Exercices de philosophie littéraire, Paris, PUF, « Pratiques théoriques », 1990, chap. 4.

31. . AlexandreкојÈVE, « Les romans de la Sagesse », Critique, mai 1952, p. 388.

32. . AlexandreкоJÈVE, art. cit., p. 389.

33. . Raymond QUENEAU, Le Dimanche de la vie, Paris, Gallimard, 1952 ; rééd. Folio, p. 206.

34. . "C'est le dimanche de la vie, qui nivelle tout et éloigne tout ce qui est mauvais; des hommes doués d'une aussi bonne humeur ne peuvent être foncièrement mauvais ou vils ». Cette citation de Hegel est reprise par Kojève en tête de son article consacré aux romans de Queneau.

35. .RaymondQUENEAU, op. cit., p. 245.

36. .Ibid., p. 234.

37. . Alexandre KoJÈVE, art. cit., p. 394-395.

\section{RÉSUMÉS}

Dans cet article, nous nous efforçons de circonscrire le champ de problèmes à partir duquel Bataille et Queneau peuvent être situés l'un par rapport à l'autre, mais aussi l'un et l'autre par rapport à Kojève et à sa lecture de Hegel dans les années 1930. Ce champ problématique apparaît polarisé par les thématiques du Désir et de l'Histoire. À partir de là, nous montrons comment les spéculations philosophiques et littéraires de Bataille sur l'expérience-limite le conduisent à dédialectiser et, finalement, à détéléologiser le Désir et à substituer à la perspective kojévienne d'une Sagesse post-historique le thème d'un érotisme transgressif. De son côté, Queneau offre une contestation plus nuancée du kojévisme dans le contexte de fictions littéraires où la conception kojévienne de l'Histoire se trouve ironisée, et finalement relativisée.

This paper seeks to outline the field of issues around which Bataille and Queneau can be relatively situated, as well as positioned relatively to Kojève and his reading of Hegel in the 1930s. It appears that this field has two thematic poles, history and desire. From there, we can show how Bataille's philosophical and literary speculation on the limit-experience led him first to de-dialectize and finally to de-teleologize desire, and to subsitute the theme of a transgressive eroticism to the Kojevian perspective of a post-historical "wisdom". For his part, Queneau offers a more nuanced dissent from Kojevism, with literary fictions in which the Kojevian conception of history is put into perspective through irony. 
Wie situieren sich Bataille und Queneau zueinander? Aber auch in Bezug auf das Werk Kojèves und seine Wiedergabe Hegels in den 30iger Jahren...

\section{AUTEUR \\ PHILIPPE SABOT}

Philippe Sabot, né en 1969, est Professeur de philosophie à l'Université Lille 3 et membre de l'UMR 8163 « Savoirs, textes, Langage » où il anime la thématique de recherche « Savoirs et pratiques de pensée : la philosophie française contemporaine ». Ses travaux portent notamment sur les rapports entre philosophie et littérature (en France, au xx siècle), sur la pensée de Michel Foucault, sur divers aspects du post-hégélianisme (en Allemagne et en France). Il a notamment publié Pratiques d'écriture, pratiques de pensée. Figures du sujet chez Breton/Éluard, Bataille et Leiris (Presses Universitaires du Septentrion, 2001), Philosophie et littérature. Approches et enjeux d'une question (PUF, 2002), Littérature et guerres. Sartre, Malraux, Simon (PUF, 2010) ; Lire Les Mots et les Choses de Michel Foucault (PUF, 2006); Ludwig Feuerbach. L'essence du christianisme (Ellipses, 2000), Héritages de Feuerbach (Ph. Sabot, dir.) (Presses Universitaires du Septentrion, 2008). Il a également traduit Judith Butler, Sujets du désir (PUF, 2011). 